\title{
Refractive Index Imaging of Cells with Variable-Angle Near-Total Internal Reflection (TIR) Microscopy
}

\author{
Kevin P. Bohannon, ${ }^{1}$ Ronald W. Holz, ${ }^{1}$ and Daniel Axelrod ${ }^{2, *}$ \\ ${ }^{1}$ Department of Pharmacology, University of Michigan Medical School, Ann Arbor, MI 48109, USA \\ ${ }^{2}$ Departments of Physics and LSA Biophysics, University of Michigan, Ann Arbor, MI 48109, USA
}

\begin{abstract}
The refractive index in the interior of single cells affects the evanescent field depth in quantitative studies using total internal reflection (TIR) fluorescence, but often that index is not well known. We here present method to measure and spatially map the absolute index of refraction in a microscopic sample, by imaging a collimated light beam reflected from the substrate/buffer/cell interference at variable angles of incidence. Above the TIR critical angle (which is a strong function of refractive index), the reflection is $100 \%$, but in the immediate sub-critical angle zone, the reflection intensity is a very strong ascending function of incidence angle. By analyzing the angular position of that edge at each location in the field of view, the local refractive index can be estimated. In addition, by analyzing the steepness of the edge, the distance-to-substrate can be determined. We apply the technique to liquid calibration samples, silica beads, cultured Chinese hamster ovary cells, and primary culture chromaffin cells. The optical technique suffers from decremented lateral resolution, scattering, and interference artifacts. However, it still provides reasonable results for both refractive index ( 1.38) and for distance-tosubstrate $(\sim 150 \mathrm{~nm})$ for the cells, as well as a lateral resolution to about $1 \mu \mathrm{m}$.
\end{abstract}

Key words: index of refraction, total internal reflection, microscope optics, cellular imaging, evanescent wave

\section{INTRODUCTION}

We here demonstrate a conceptually simple method for recording spatially-resolved maps of the absolute refractive index of living cells adhered to a coverslip surface. The approach also yields, as a separate result, the local distance between the bottom of the cell and the coverslip. The technique is nonperturbative, requires no labeling, uses only one illumination wavelength and requires only the standard cell buffer medium without replacement. The technique's optical setup can be completely compatible to that for objectivebased total internal reflection fluorescence (TIRF), such that the same field of view through the same objective on the same sample can be examined, and produces a lateral resolution down to about $1 \mu \mathrm{m}$.

Measurement of the spatially localized refractive index within the cell cytoplasm has several applications:

(a) In the TIRF technique, which is useful for examining intracellular processes near cell/substrate contact regions, the depth of the evanescent field $d$ (the "penetration depth") depends on the cytoplasmic refractive index $n_{1}$ (Axelrod, 2013). Some TIRF applications (such as observation of the axial motion of organelles) can provide correct quantitative results only with an accurate value for $d$. (Steyer et al., 1997; Holz \& Axelrod, 2008).

(b) The visual systems in a variety of organisms depend on spatially varying $n_{1}$ (Axelrod et al., 1988).

Received May 19, 2017; accepted August 5, 2017

*Corresponding author. daxelrod@umich.edu (c) The spatially resolved local index of refraction can be a measure of the local protein concentration.

(d) Cellular pathophysiology, e.g., transformation to cancer (Backman et al., 2000; Popescu, 2011; Lee et al., 2013; Liu et al., 2016), is sometimes accompanied by alterations in $n_{1}$, which can thereby be used as an optical microscopy indicator.

(e) Local refractive index can affect fluorescent lifetimes (Tregidgo et al., 2008). This effect can be used to better interpret FLIM images and also provide a means to report local refractive indices (Tregidgo et al., 2007; Van Manen et al., 2008).

Measurement of the distance $h$ from the coverslip surface to the plasma membrane of the cell also has applications.

(a) In principle, the distance $h$ can report the thickness of the collagen layer on coated coverslips. In practice, to obtain this information with the present technique, we would also need to know the refractive index of the collagen, which can vary with density.

(b) The micromorphology of the bottom of the cell may be related to sites of exocytosis and endocytosis. The present technique requires the cell bottom to be within a wavelength or so from the substrate; this appears to be the case based on the visibility of the plasma membrane of secretory cells under TIRF illumination (Anantharam et al., 2012).

(c) The intensity of TIRF at any location results from a combination of local fluorophore concentration and position in the evanescent field; measurement of local $h$ can resolve the ambiguity and thereby lead to a more accurate picture of the local concentration. 
A sample's local refractive index is the key to several microscope imaging techniques. Some techniques that use transmitted illumination convert refractive index gradients to image plane intensity variations on samples that are inherently clear and colorless: e.g., phase contrast, differential interference contrast, and dark field. Other techniques qualitatively image different refractive index zones by detecting deflection of the transmitted beam direction, e.g. modulation contrast (Hoffman \& Gross, 1975; Axelrod, 1981; Mitsui, 2005). But these techniques have not been set up to report absolute refractive index.

Other techniques depend on interference between a sample-transiting beam and a reference beam of partially coherent light to produce a quantitative measure of local optical path length (a product of refractive index and sample thickness): e.g., quantitative phase microscopy (QPM) (Curl et al., 2005; Choi et al., 2007; Jenkins \& Gaylord, 2015). Differential interference contrast images can also be mathematically analyzed to produce quantitative measures of phase retardation due to optical path length (Kou et al., 2010). It is possible to separate out the refractive index factor from the thickness factor, but it can be complicated. Some approaches to this separation necessitate the use of two colors (Rappaz et al., 2005, 2008; Jafarfard et al., 2014; Friedman \& Shaked, 2015), variation of the refractive index of the surrounding medium (Ross, 1954; Rappaz et al., 2005, 2007), simplifying geometrical assumptions (Martinez-Torres et al., 2015), a combination of QPM with confocal microscopy (Curl et al., 2005) or single-point measurements (Friedman \& Shaked, 2015). Quantitative measurements of changes in cell volume can be derived from optical path length changes, as measured by phase contrast and dark-field images (Farinas et al., 1997). Comparison of images in focus and slightly out-of-focus also provides some information about refractive index, either in QPM with a reference beam (Jenkins \& Gaylord, 2015) or in bright field (Phillips et al., 2012; Neto et al., 2016).

Reflection, rather than transmission, microscopy has also been used to measure refractive index in the cell zone nearest to the substrate, so cell thickness is not a factor there. But even with reflection, analysis is still complicated by the presence of an intermediate layer (media or collagen) of thickness $h$ between the substrate and the cell. Interference reflection contrast (IRC) microscopy detects the interference between light reflected from the substrate/intermediate layer and light reflected from the intermediate layer/cell surface. Because of the typical $180^{\circ}$ phase shift at the latter reflection, the intensity of the combined reflection decreases with decreasing $h$. Close apposition regions look the darkest and exactly how dark depends upon the amplitude of the intermediate layer/cell surface reflection, and that amplitude depends upon the local cell surface refractive index. Usually, IRC is used as a qualitative technique, but by using two different wavelengths or two different apertures, quantitative values for refractive index and intermediate layer thickness can be deduced (Bereiter-Hahn et al., 1979). Other reflection (e.g., surface plasmon resonance imaging, Yanase et al.,
2010) or back-scattering techniques (Cherkezyan et al., 2014) have also been used to measure refractive index or its fluctuation rates.

Here we present a relatively direct method for obtaining spatial maps of the local refractive index of cells: measuring the position of the reflected intensity versus incidence angle curve. This position reveals the critical angle for total internal reflection, which depends strongly upon cell refractive index $n_{1}$. The shape of the reflected intensity versus incidence angle curve depends strongly on the intermediate layer thickness $h$. From the critical angle (affected most by $n_{1}$ ) and the shape (affected most by $h$ ) of the reflected intensity versus incidence angle curve at each pixel in the image, absolute values for $n_{1}$ and for $h$ can be calculated and plotted as a spatial map. This technique requires neither double wavelengths nor refractive index alterations in the medium. The optical configuration can be very similar to standard objective-based TIRF (except without the emission barrier filter) and could easily be used on exactly the same sample in the course of a TIRF experiment. But like most of the above-discussed techniques, it requires several images to be taken (here at different incidence angles) as well as some post-image processing. The main drawback of the present technique is a loss of optimal resolution, because the full aperture of the objective is not used for light collection. Variable angle TIR has been used previously, typically for changing the depth of the evanescent field in fluorescence applications (Oheim et al., 1999; Loerke et al., 2000; Sun et al., 2011).

We first review the theory, all of which is well-known in classical optics. This is followed by descriptions of the optical configuration; a simplified curve fitting procedure for reflected intensity at each pixel versus incidence angle; and the essential calibration procedure on known refractive index liquids. Then the technique is applied to "unknown" samples of cultured Chinese hamster ovary $(\mathrm{CHO})$ cells and primary bovine adrenal chromaffin cells, and a verification on spherical silica beads.

\section{THEORY}

Figure 1 defines the variables. A collimated light incident beam of unit intensity travels from the glass substrate (refractive index $n_{3}$ ) at incidence angle $\theta_{3}$, where it encounters a water or collagen intermediate layer (refractive index $n_{2}$ ) of thickness $h$, and then encounters the cell (refractive index $n_{1}$ ). The intensity $I$ of the reflection of the layered system back into $n_{3}$ (normalized to the incident intensity) is measured as a function of $\theta_{3}$. The following expressions are derived in Born \& Wolf (1975) but here with notational alterations and with subscripts 1 and 3 reversed to conform to the conventions of Hellen \& Axelrod (1987):

$$
I=\left|\frac{r_{32}+r_{21} e^{2 i \beta_{2}}}{1+r_{32} r_{21} e^{2 i \beta_{2}}}\right|^{2}
$$




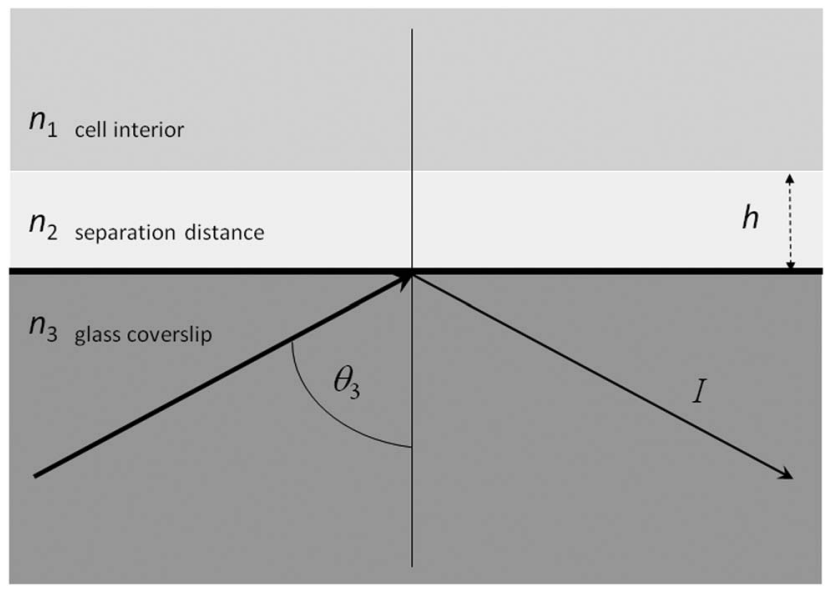

Figure 1. Definition of optical variables at an idealized cell/glass substrate contact region with a separation $h$. The reflected intensity is for the whole system, including the effects of both interfaces.

Variables $r_{i j}$ are the single-interface Fresnel reflection coefficients for light traveling from $n_{i}$ to $n_{j}$. These depend on the polarization $\mathrm{s}$ - or $\mathrm{p}$ - of the incident light:

$$
\begin{aligned}
& \text { for s-pol: } r_{i j}=\frac{n_{i} \cos \theta_{i}-n_{j} \cos \theta_{j}}{n_{i} \cos \theta_{i}+n_{j} \cos \theta_{j}} \\
& \text { for p-pol: } r_{i j}=\frac{n_{j} \cos \theta_{i}-n_{i} \cos \theta_{j}}{n_{j} \cos \theta_{i}+n_{i} \cos \theta_{j}},
\end{aligned}
$$

where, from Snell's law and trigonometry,

$$
\begin{aligned}
& \sin \theta_{k}=\left(n_{3} / n_{k}\right) \sin \theta_{3} \\
& \cos \theta_{k}=\sqrt{1-\sin ^{2} \theta_{k}},
\end{aligned}
$$

where $k=1,2$, or 3 and $\theta_{k}$ is the angle of the ray relative to the normal in each medium. Variable $\beta_{2}$ contains all the dependence upon $h$ :

$$
\beta_{2}=2 \pi n_{2}(h / \lambda) \cos \theta_{2} .
$$

For unpolarized or circularly polarized incident light, the intensity is just the average of the p-pol and s-pol $I$, as calculated above.

The incidence angle $\theta_{3}$, as measured in $n_{3}$, is always real, as is $\cos \theta_{3}$ and $\sin \theta_{k}$. However, $\cos \theta_{1}$ and/or $\cos \theta_{2}$ can be imaginary above some critical angle for total internal reflection, for sufficiently large $\left(n_{3} / n_{1}\right)$ or $\left(n_{3} / n_{2}\right)$.

Figure 2a shows the theoretical polarized $I$ versus $\theta_{3}$ for a range of $n_{1}$ values chosen to match particular water/ glycerol mixtures to be tested (as discussed below), always with $h=0$. Each of this set of curves shows a steep rise up to the TIR critical angle at which $I$ achieves a value of 1 ; the effect of increasing $n_{1}$ is mainly a shift toward higher $\theta_{3}$ while maintaining the steep shape. Of course, with $h=0$, then $\beta_{2}=0$ and $I$ versus $\theta_{3}$ is unchanged, regardless of the value of $n_{2}$ as expected.

Figure 2b shows unpolarized $I$ versus $\theta_{3}$ for a particular single $n_{1}$ but with a range of intermediate layer $h / \lambda$ values. The effect of $h>0$ is to broaden $I$ versus $\theta_{3}$ so it starts rising at smaller $\theta_{3}$ while still achieving TIR at the same critical
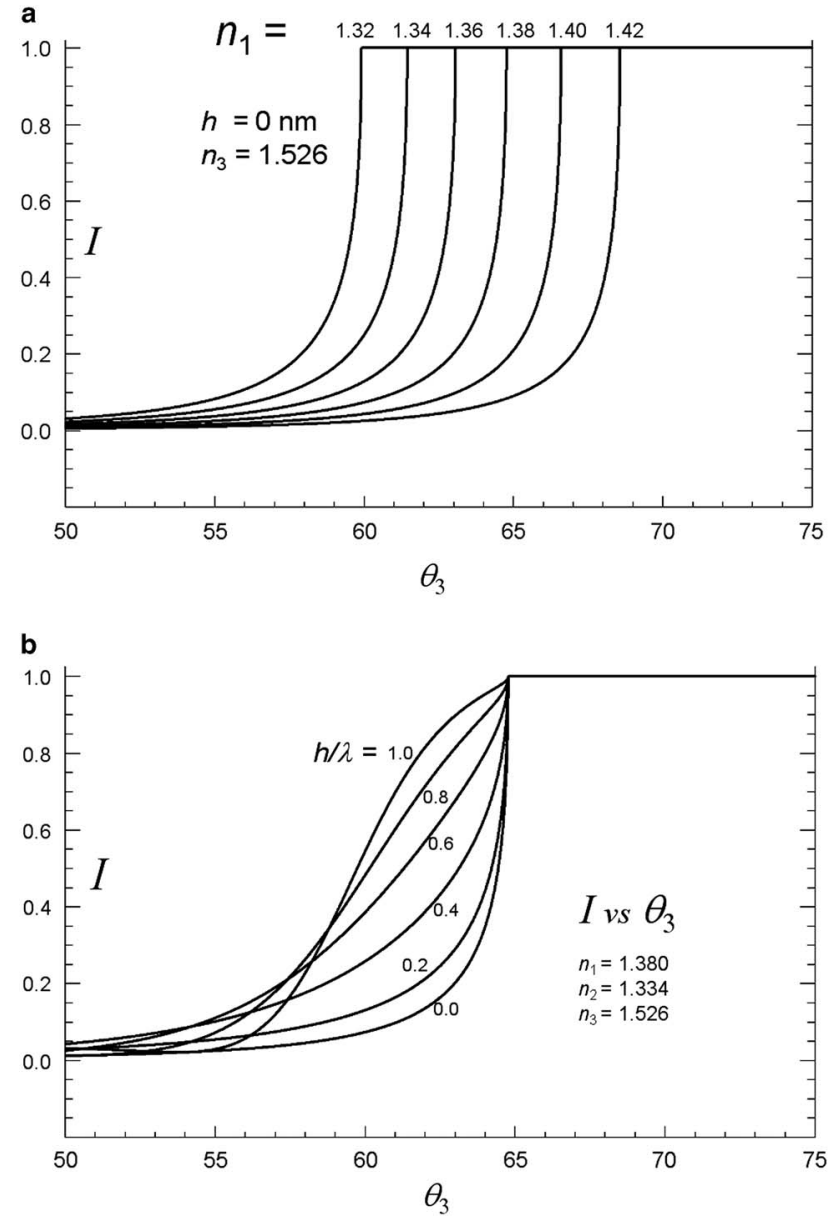

Figure 2. Normalized reflected intensity $I$ versus incidence angle $\theta_{3}$ for (a) a range of $n_{1}$, all with $h=0$; and (b) a single $n_{1}$ but with a range of $h$ values. Unpolarized light is assumed.

angle. The clear distinction between the different effects of $n_{1}$ and $h$ upon $I$ versus $\theta_{3}$ is the basis for distinguishing $n_{1}$ and $h$. Of course, if $n_{2} \sim n_{1}$, then the term "intermediate layer" has little meaning, and varying $h / \lambda$ has little effect.

\section{Methods}

\section{Optics}

Figure 3 shows the optical configuration for the experiments. The goal is to focus a laser beam at the objective's back focal plane (BFP) so the beam emerges in collimated form at a controllable angle. The polar angle of incidence $\theta_{3}$ (measured relative to the optical axis) is varied by adjusting the radial position of the BFP focus with a pair of galvanometer ("galvo") mirrors (Thorlabs) driven by analog voltages generated by a National Instruments 6353 I/O interface card controlled by a custom LabView program. The optical configuration is based upon a custom objective-based ("through-the-lens") TIRF system (using an Olympus IX-70 inverted microscope with a NA $1.4960 \times$ objective), but with the standard fluorescence dichroic mirror/barrier filter cube removed and replaced with a fully reflective 


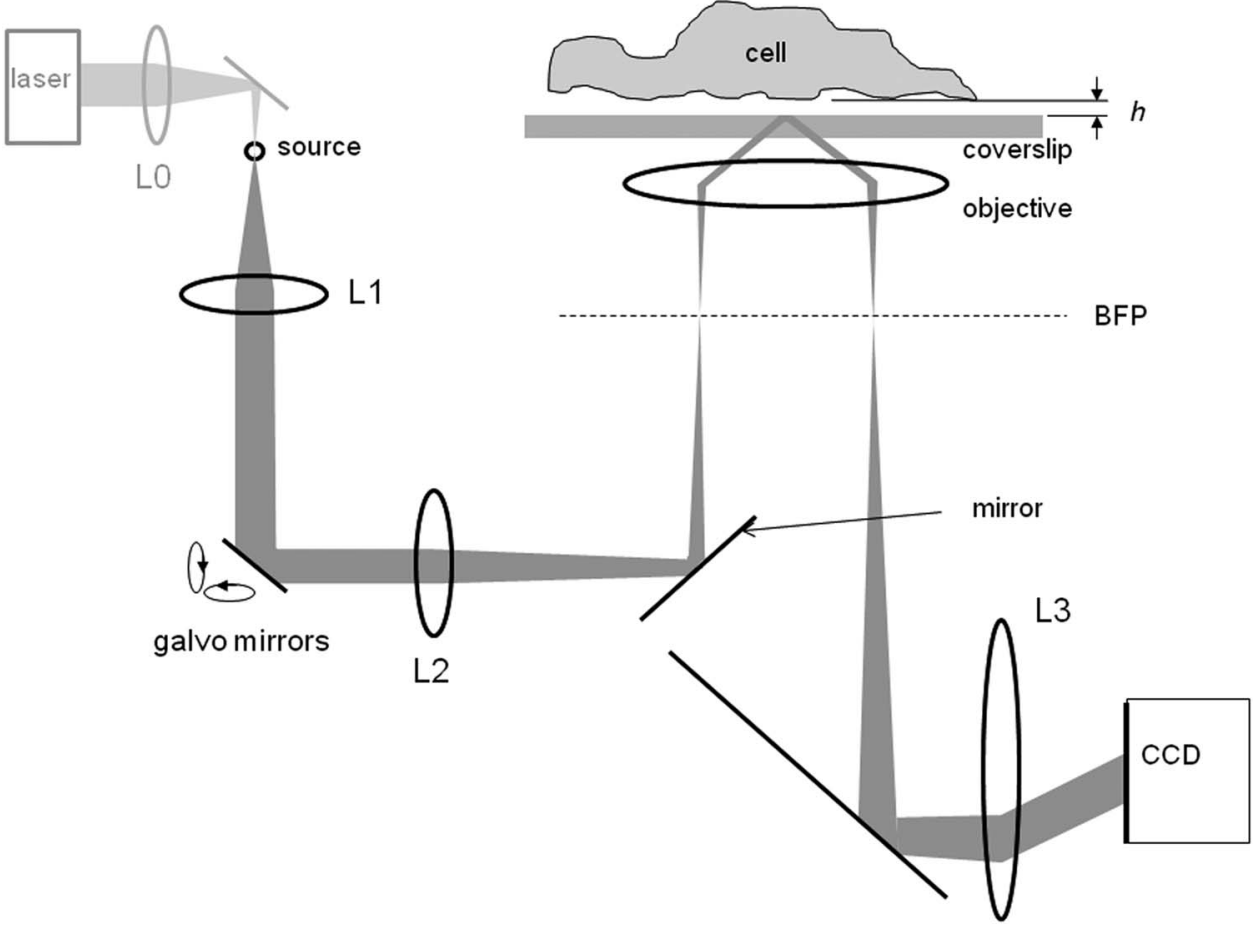

Figure 3. The optical configuration for the experiments. The "source" can be either a small LED or the position of a laser beam focus (produced by lens L0, shown in gray). Lens L1 collimates the source; selection of its position and focal length allows for setting the desired width of the collimated beam (and ultimately the area of illumination on the sample). The galvo mirrors are placed close to a plane complimentary to the sample plane. Lens L2 (focal length $150 \mathrm{~mm}$ here) refocuses the beam to the back focal plane of the high aperture (NA 1.49) objective at an off-axis radial position, adjustable by programming one of the galvo mirrors. In principle, the focus at the BFP ensures that the emerging beam is collimated; in practice it diverges with a half angle of about $1-2^{\circ}$ due to aberrations in the objective periphery. A $100 \%$ reflecting mirror (in place of the standard dichroic mirror used for fluorescence) is positioned $15-30 \%$ across the aperture to direct the incident light up toward sample. Lens L3 recollimates the reflected beam and casts it upon a CCD array positioned at a plane complimentary to the sample plane. The distance scales are distorted for pictorial clarity.

mirror (and no barrier filter) that only extends a small fraction (15-30\%) across the aperture from one side. This ensures that the incident beam deflects up into the objective (and focuses at the BFP) but the reflected beam from the sample passes back down on the opposite side of the aperture unimpeded. This configuration prevents light scattered from the first deflection of the beam toward the sample from reaching the camera, but in our implementation, the incident beam approaches the sample from one azimuthal angle only.

A diode laser beam (coherent, $561 \mathrm{~nm}$ ) is used as the source here. A narrow band LED source could also be used; it would have the advantage that interference fringe effects would be somewhat reduced (but not entirely eliminated).

A sequence (or "stack") of images is recorded: each frame in the sequence corresponds to a small step increase in polar incidence angle $\theta_{3}$.

\section{Samples}

A sequence of reflected light images at incremented incidence angles is recorded on four types of samples: (a) liquids of known index of refraction to calibrate the $\theta_{3}$ axis; (b) nonconfluent cultured $\mathrm{CHO}$ cells; (c) primary chromaffin cells adhered to glass; and (d) spherical silica beads of 20- $\mu \mathrm{m}$ diameter and index $n_{1}=1.46$ (Corpuscular, Inc., Cold Spring, NY, USA) to verify the technique for deducing the varying depth $h$ of the intermediate layer of water between the substrate surface and the silica bead. Bovine chromaffin cells (without differential plating) were prepared as in Wick et al. (1993). The CHO cells were obtained from the laboratory of Dr. John Traynor. None of these samples are labeled with chromophores or fluorophores.

The liquids of known refractive index were freshly prepared glycerol water mixtures using freshly opened glycerol bottles, in measured weight-to-weight proportions. To avoid refractive index instabilities due to the hygroscopicity of glycerol, the index of refraction of each mixture was confirmed with a standard refractometer shortly before each experiment. The measured values always agreed with the expected published values.

All of the samples (liquids, cells, and beads) were contained in commercial 1.5-thickness glass coverslip-bottomed 35-mm diameter culture dishes (FluoroDishes; World Precision Instruments, Inc., Sarasota, FL, USA). The entire field of view appeared in focus, so the coverslip was deemed 
to be horizontal to within a couple tenths of degrees of tilt at most.

\section{Normalization of $\boldsymbol{I}$}

\section{Highest Value Reset}

Experimental images have interference fringes and artifactual shadows as the incident beam approaches and exceeds TIR critical angle. This is especially true on samples with variegated refractive index (such as cells). The fringes and shadows cause somewhat noisy or undulating $I$ versus $\theta_{3}$ curves [either in large regions-of-interest (ROI) or in single pixels], which make it difficult to determine where $100 \%$ reflectivity occurs. In uniform samples (such as liquids used for calibration), the undulations are not severe. As $\theta_{3}$ increases for such samples, the first-achieved local maximum in $I$ is normalized to 1 , and all values of $I$ at higher $\theta_{3}$ are reset to 1 . In nonuniform samples, such as cells or beads, the undulations are more severe at high $\theta_{3}$. For such samples, the absolute maximum intensity in each $I$ versus $\theta_{3}$ curve is located but then the data were normalized to its value where it first crosses a fraction $\gamma$ (set equal to 0.6 here) of that maximum intensity. All intensity values at higher $\theta_{3}$ are reset equal to 1.

To show how the normalization is applied, Figure 4 shows typical $I$ versus $\theta_{3}$ curves from two different pixels on an actual $\mathrm{CHO}$ cell image stack, one on-cell and one off-cell. In theory (see Fig. 2b), $I$ versus $\theta_{3}$ curves (for $h<\lambda / 2$ ) are the steepest as $\theta_{3}$ closely approaches the critical angle $\theta_{c}$ for TIR and above which $I$ versus $\theta_{3}$ is flat at 1 (apart from a slight rounding due to a spread of angles in the incident beam). In practice, the experimental normalized $I$ versus $\theta_{3}$ curves can be noisy, displaying undulations due to scattering and coherent light interference effects. But even in highly scattering samples, a very steep section almost always appears in the experimental $I$ versus $\theta_{3}$ curves. The $\theta_{3}$ location of the top of that steep section is assumed to correspond to the TIR critical angle. In our data, we find that setting $\gamma$ at 0.6 successfully locates the approximate top of the steep section in almost all the pixels of an image stack, while truncating off the spurious undulations. To suppress small rapid fluctuations (probably due to shot noise and camera noise), the $I$ versus $\theta_{3}$ are presmoothed by a traveling average of "boxcar" width of five data points before further analysis.

\section{Baseline Reset}

Because there is considerable scattering of the incident light at all surfaces in the objective and the sample (and there is no barrier filter to exclude it), the reflected beam is the major, but not the only, part of the total light seen by the camera. Therefore, the correct "zero" baseline for just the reflected beam is difficult to determine from experimental data. However, we know the theoretically expected value for $I$ value at every incidence angle for each calibration liquid $n_{1}$, so we adjust the baseline on the experimental data to give the

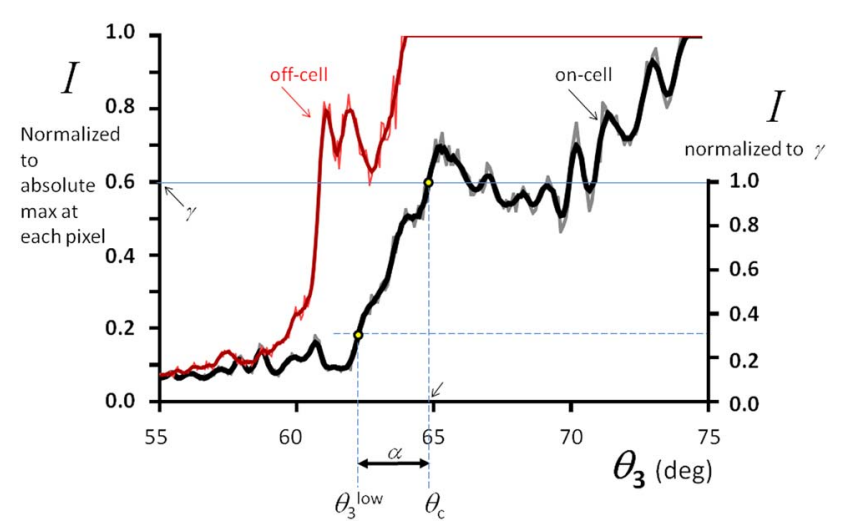

Figure 4. Intensity normalization and deduction of $n_{1}$ and $h$, illustrated on actual $I$ versus $\theta_{3}$ curves taken from an on-cell pixel (black lines) and off-cell pixel (red lines) at a Chinese hamster ovary cell. All measurements are taken on the smoothed graphs (heavier lines) rather than the noisier raw data (lighter lines). The left $I$ axis is normalized to the absolute maximum in the measured $I$ versus $\theta_{3}$ curve at each pixel independently, regardless of whether the pixel is on-cell or off-cell. Therefore, the intensity scale shown on the left $I$ axis is different for different pixels, and " 1 " corresponds to different absolute intensities for different pixels. The effective "100\%" reflection level is defined to be a fraction $\gamma$ (here set at 0.6 ) of the normalized maximum in $I$, The $\gamma$ renormalized $I$ scale is shown as the right-side axis. Angle $\theta_{c}$ occurs at the smallest angle where the smoothed data crosses the renormalized intensity of 1.0. Angle $\theta_{3}^{\text {low }}$ occurs where the smoothed data crosses the renormalized intensity of 0.3 (close to the bottom of the steep rise for almost all pixels). Note that the on-cell steep slope section occurs at a higher angle than the offcell steep slope section, indicative of the higher on-cell refractive index. Also note that the steep slope section for the on-cell curve is considerably less steep than the corresponding slope of the offcell curve, indicative of the larger $h$ at the on-cell location.

correct value at the lowest incidence angle. Light scattering by cells can be a function of the observation angle (McManus et al., 1993), but the scattered light here is gathered by the objective (and thereby effectively averaged) over a wide range of angles. Therefore, we assume that the scattering background power is not nearly as strong a function of $\theta_{3}$ as the reflected beam power.

\section{Calibration of $\boldsymbol{\theta}_{\mathbf{3}}$}

The relationship between voltage $V$ to the galvo mirror and angle $\theta_{3}$ must be established to properly scale the abscissa of the $I$ versus $\theta_{3}$ curves. $V$ is incremented by constant steps. But $\theta_{3}$ is not measured directly, and the relationship between $V$ and $\theta_{3}$ may not be linear. The nonlinearity of $V$ versus $\theta_{3}$ is measured by coupling the incident beam out of the objective with a glass prism, measuring the position of resulting laser illumination on the wall, and back-calculating $\theta_{3}$ using Snell's law. A full spread of about $2^{\circ}$ of incidence angle is manifested by a vertical streak rather than a spot on the wall. This streak makes absolute measurement of $\theta_{3}$ somewhat ambiguous, but the change of streak position with an increment in $V$ can be measured more 
accurately. Given this data, the nonlinear relationship between galvo voltage and $\theta_{3}$ can be established and fit to a fifth-order polynomial for convenience.

As can be seen from Figure 2a, the reflected intensity is extremely sensitive to the theoretical $\theta_{3}$. But the actual experimental $\theta_{3}$ at any particular $V$ is also very sensitive to the optical alignment, so a small error in estimation of $\theta_{3}$ can lead to a large error in predicted intensity. To get around this inherent problem of consistent and reproducible calibration, we compare $I$ versus $\theta_{3}$ curves from an unknown sample (or single pixel data in an image stack) to those of liquids with known refractive indices, to find a single calibration scale for the angles $\theta_{3}$ that then can be applied to any data on an unknown sample. These liquids consist of series of glycerolwater mixtures. Using the average intensity versus frame number within a large ROI on a known liquid, we adjust the position and scale of the experimental curve's abscissa (the $\theta_{3}$ axis) so that the experimental average $I$ versus $\theta_{3}$ curves obtained for the lowest index $\left(n_{1}=1.334\right.$, water $)$ and highest index $\left(n_{1}=1.412,60 \%\right.$ glycerol:water, w/w) match their corresponding theoretical curves (for $h=0$ ). We can then test whether experimental $I$ versus $\theta_{3}$ curves for intermediate refractive index liquids fall in the correct position on this $\theta_{3}$ axis, as calculated from theory, as discussed in the beginning of the Results section.

\section{Analyzing $I$ Versus $\theta_{3}$ to Obtain $\boldsymbol{n}_{\mathbf{1}}$ and $\boldsymbol{h}$}

The intensity is determined either (a) over a large ROI for calibrations using glycerol-water liquid samples (where no intermediate layer exists, i.e., $h=0$ ), or (b) pixel-by-pixel for samples where spatially varying refractive indices and intermediate layer thicknesses are expected.

The scheme for determining $n_{1}$ and $h$ is depicted in Figure 4, using, as an example, an actual $\mathrm{CHO}$ cell culture $I$ versus $\theta_{3}$ curve at a single on-cell pixel (black curve). Rather than doing a full-scale least-squares minimization curve fitting of the normalized $I$ versus $\theta_{3}$ at each pixel of a sample image (which can be very time-consuming on the computer), we can quickly estimate $n_{1}$ and $h$ at each pixel by measuring the two angles $\theta_{3}^{\text {low }}$ and $\theta_{c}$ for which $I\left(\theta_{3}^{\text {low }}\right)=0.3$ and $I\left(\theta_{c}\right)=1.0$. Refractive index $n_{1}$ can be deduced directly from the relationship $n_{1}=n_{3} \sin \theta_{c}$, where the substrate refractive index $n_{3}$ here is 1.5255 . For $h<\lambda / 2, \theta_{c}$ should be insensitive to changes in $h$ (as can be seen in Fig. 2b).

On the other hand, the difference $\alpha=\theta_{c}-\theta_{3}^{\text {low }}$ is always a monotonically increasing function of $h$ in the range of $h<\lambda / 2$. However, $\alpha$ does depend somewhat on $n_{1}$. A complete set of theoretical curves can be generated and analyzed to show how the theoretical $\alpha$ depends upon $n_{1}$ and $h$. Given any experimental $n_{1}$, as deduced from $\theta_{c}$, a theoretical curve of $\alpha$ versus $h$ can be generated. From the experimentally measured $\alpha$, the corresponding experimental $h$ can be located unambiguously from the $\alpha$ versus $h$ graph.

All of the computations on image stacks were done with a software program custom written in Interactive Data Language (IDL).

\section{Reflected intensity vs incidence angle} for calibration liquids

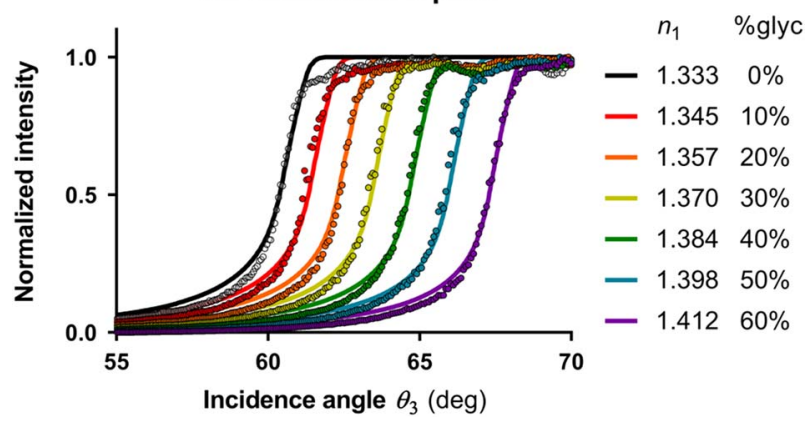

Figure 5. Experimental $I$ versus $\theta_{3}$ curves for glycerol:water calibration liquids of the indicated $n_{1}$ values (discrete data points), along with theoretically generated curves (solid lines) for that known $n_{1}$ and $h=0$. The intensities were gathered from a large region of interest rather than a single pixel. The theta scale is adjusted so the experimental and theoretical curves agree at the lowest ( $0 \%$ glycerol) and the highest (60\%) samples. For the intermediate index calibration liquids, the experimental curves agree well with the theoretical predictions. The spread of incidence angles in the actual experiment is simulated by a Gaussian convolution averaging along each theoretical curve with a full width of 1.0 degrees in incidence angle; this is why the total internal reflection critical angle does not produce a sharp corner in the curves as they achieve their maximum value of 1 .

\section{Results}

\section{Glycerol: Water Mixtures, Large ROI}

Figure 5 shows $I$ versus $\theta_{3}$ curves from a large ROI on each glycerol:water calibration liquid $(0,10,20,30,40,50$, and $60 \%)$ and a set of theoretically generated curves (including smoothing by a Gaussian kernel convolution to account for a $1^{\circ}$ spread) for the (known) $n_{1}$ values of the experimental liquids. Although the match is not surprising for the lowest and highest $n_{1}$ indices (because the $\theta_{3}$ calibration was deliberately adjusted to achieve that match), the intermediate $n_{1}$ curves also show good agreement between the actual $n_{1}$ and the theoretical prediction for that $n_{1}$. This confirms that the calibration of galvo mirror voltage $V$ with $\theta_{3}$ is correct over the entire range of expected $n_{1}$ values. There are some small undulations (rather than flat at 1.0) in the experimental $I$ versus $\theta_{3}$ curves even where TIR has already been achieved because of scattering and interference artifacts, but these are not as severe here as in the spatially heterogeneous cell and bead samples considered later. In view of this relatively wellbehaved experimental data, $I$ is normalized to its values at the first maximum for each calibration liquid sample.

\section{Chromaffin Cells, Large ROI}

Figure 6 shows $I$ versus $\theta_{3}$ curves for each of many chromaffin cells. The intensities were gathered from large ROI, always entirely within the cells' footprints. Although there is cell-to-cell variability, they cluster around the theoretical curve (shown in red) with $n_{1}=1.38$ (ranging from 1.37 to 1.39) and $h=150 \mathrm{~nm}$. 


\section{CHO Cells and Chromaffin Cells, Spatial Maps of $n_{1}$ and $h$}

Figure 7 shows reflected light images, selected from an image sequence for (top) a nonconfluent $\mathrm{CHO}$ cell and (bottom) a chromaffin cell adhered to the substrate, preceded by a transmitted light view to show where the cells are located. At low $\theta_{3}$, the reflection is subcritical everywhere so the image is fairly dark. At somewhat higher $\theta_{3}$, the off-cell (water) regions have achieved full TIR (and appear bright) but the on-cell regions are still subcritical (and appear relatively dark). At the highest $\theta_{3}$, the entire field of view is supercritical and thereby appears bright everywhere.

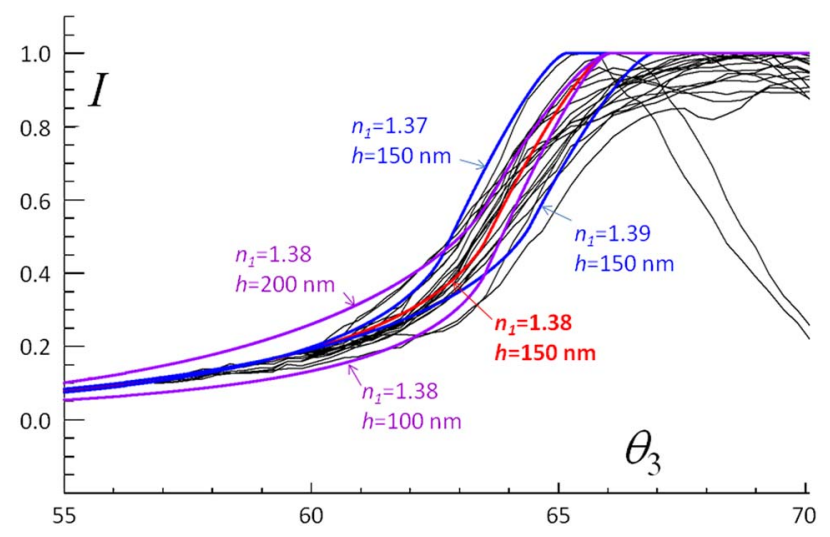

Figure 6. Experimental $I$ versus $\theta_{3}$ curves for 22 different chromaffin cells (black lines). The intensities were gathered from a large regions of interest, always entirely within the cells' footprints. Although there is cell-to-cell variability, they cluster around a theoretical curve (shown in red) with $n_{1}=1.38$ and $h=150 \mathrm{~nm}$. Theoretical curves with $n_{1}=1.37$ or 1.39 and $h=150 \mathrm{~nm}$ (blue) are significantly different from the center of the experimental cluster. Theoretical curves with $n_{1}=1.38$ and either $h=100$ or $200 \mathrm{~nm}$ also do not follow the experimental cluster. These theoretical curves assume a spread in incidence angle of $2.0^{\circ}$.

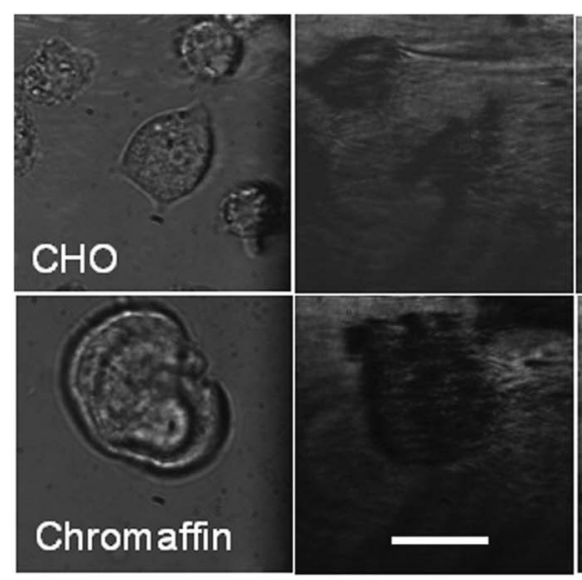

$\theta_{3}=$ $59.9^{\circ}$

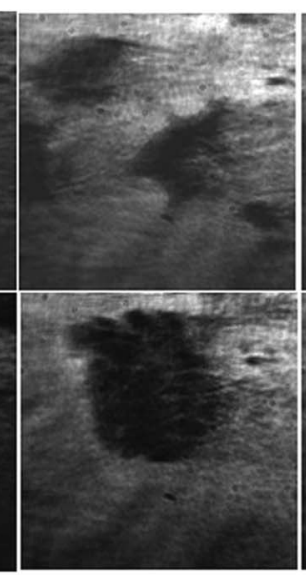

$61.2^{\circ}$

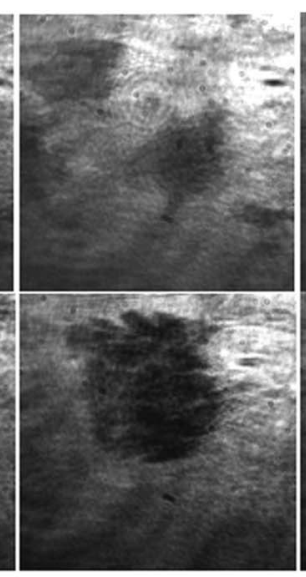

$62.6^{\circ}$

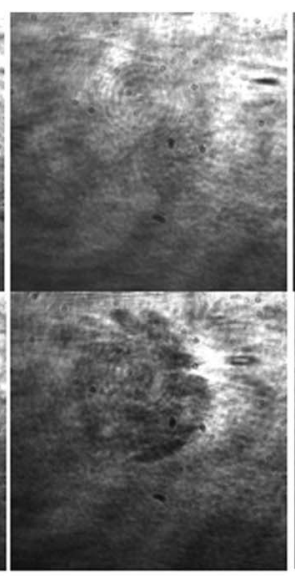

$64.1^{\circ}$

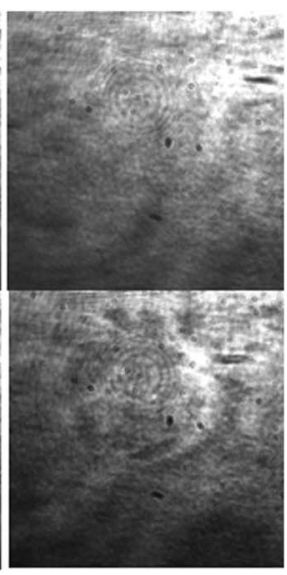

$65.6^{\circ}$

Figure 7. Raw reflected light images from an image sequence for Chinese hamster ovary (CHO) cells (top row) and chromaffin cells (bottom row) with selected increasing $\theta_{3}$. A transmitted light view precedes each sequence to show where the cells are located. The images are cropped to $800 \times 800$ pixels from the original $2 \mathrm{~K} \times 2 \mathrm{~K}$. Scale bar is $20 \mu \mathrm{m}$. 

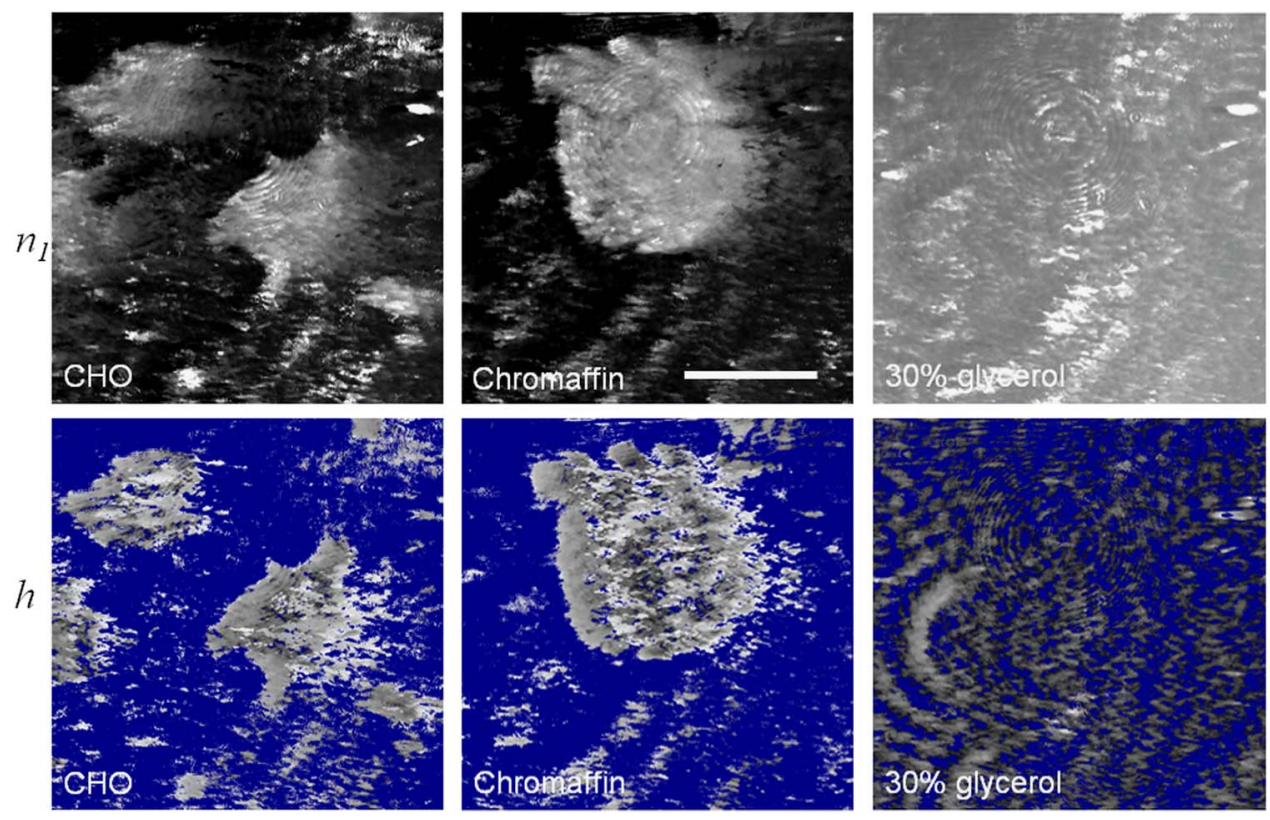

Figure 8. Calculated spatial maps for $n_{1}$ and $h$, constructed from pixel-by-pixel analysis of each $I$ versus $\theta_{3}$ curve for the exact stacks from which the Figure 7 cell sequences are sampled. The linear grayscale range for $n_{1}$ is from 1.330 (black) to 1.390 (white). All values in the $n_{1}$ less than 1.330 are shown as black; these can be as low as 1.32 due to noisy $I$ versus $\theta_{3}$ curves. The corresponding spatial maps for $h$ have a linear grayscale ranging from $10 \mathrm{~nm}$ (black) to $290 \mathrm{~nm}$ (white). The left and center column panels are for Chinese hamster ovary $(\mathrm{CHO})$ and chromaffin cells, respectively. In the off-cell regions, where $n_{1}$ is generally between 1.33 and 1.35 , theory predicts that $h$ does not strongly affect the $I$ versus $\theta_{3}$ curves. The determination of $h$ in those regions consequently is ill-defined or noisy at best. Those regions are depicted in a uniform blue. In a small minority of isolated spots at on-cell regions, calculation of $h$ (by the $\alpha=\theta_{\mathrm{c}}-\theta_{3}^{\text {low }}$ method described in the text) produced a negative $h$ due to noise and scattering in $I$ versus $\theta_{3}$ (i.e., resulting from an overly steep rise). Those pixels are assigned $h=0$. The right column panels are for a $30 \%$ glycerol:water liquid mixture, with the same grayscales as for the cells. In general, the spatial maps for liquid are more uniform than those for cells as expected, but they still do exhibit noise from interference and scattering effects. Scale bar is $20 \mu \mathrm{m}$.

similarly to the footprint of the chromaffin cell sample above gives $\left\langle n_{1}>\right.$ of 1.368 with SD of 0.006 . The $30 \%$ glycerol sample returns the expected $\left\langle n_{1}\right\rangle$.

\section{Spherical Silica Beads}

Silica beads $(20-\mu \mathrm{m}$ diameter, $n=1.46)$ were examined with variable-angle TIR for the purpose of verifying the sensitivity to predictable variations in $h$, despite the presence of scattering and interference even larger than would be expected on cells. Figure 9 (top row) shows a sequence of raw images at increasing incidence angle. Streaks of light and shadow can be seen outside on the downbeam side (the right side here) of the sphere, and this produces artifacts in both in the $n_{1}$ and $h$ spatial maps. Figure 9 (bottom row) shows those spatial maps, at a much larger magnification than the corresponding cell images in Figure 8.

Most notable is that a distinct region of close contact (small $h$ ) can be seen near the center of the contact region; it presents here as a central dark region in a grayscale that gets brighter with increasing $h$. We have no independent measurement of whether the bead is an exact sphere. The analysis procedure assigns an $h=110 \mathrm{~nm}$ at that closest approach, and $h=290 \mathrm{~nm}$, an increase of $180 \mathrm{~nm}$, in a rough ring of radius $\sim 1,500 \mathrm{~nm}$ around that spot.
Geometry suggests that such a rise should occur in a radius of $1,900 \mathrm{~nm}$.

In regions where the bead is close to the substrate (near the center of the field), TIR at the substrate/water interface is "frustrated" by the high refractive index (1.46) of the bead. Normally, incident light in that region would simply continue as propagating light away from the substrate and not be seen again. However, with a closed spherical shape, the propagating light becomes partially trapped by total reflection inside the bead, partially reemerging again near the point of substrate contact and also partially concentrating and reemerging on the downbeam side of the sphere near its equator (Chew et al., 1979). Therefore, incident light that "normally" would not be seen as a reflection back into the substrate until higher incidence angles will actually be seen at lower incidence angles. This effect will shift the deduced index $n_{1}$ to lower values than otherwise expected, an effect seen here.

\section{DISCUSSION}

Variable angle illumination in the range of incidence angles near the critical angle for TIR, along with reimaging the reflected beam, can produce information on both the refractive 


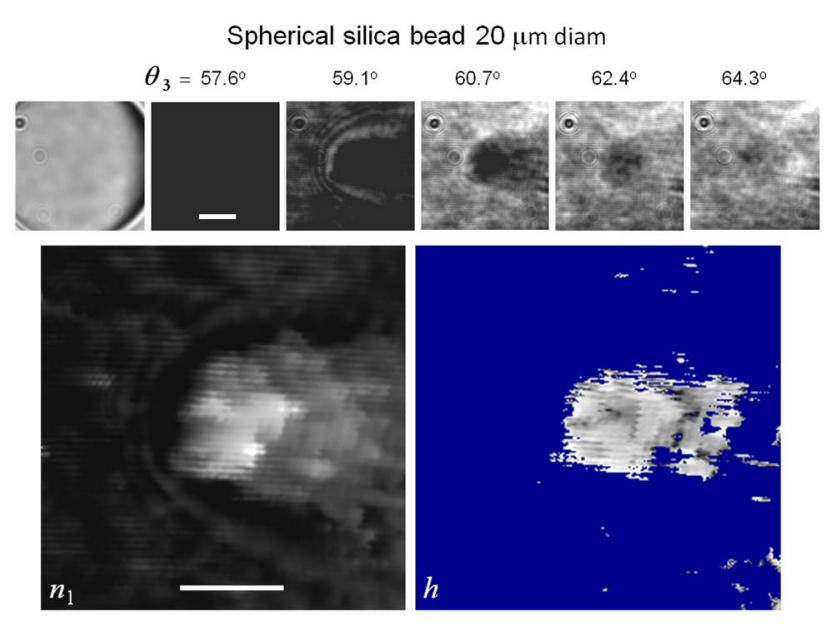

Figure 9. Top row: selected raw images of reflected light from a spherical silica bead residing on the substrate surface, as $\theta_{3}$ increases. The bead has a nominal $n_{1}=1.46$. Lower panels: spatial maps of $n_{1}$ and $h$, constructed from pixel-by-pixel curve fitting of $I$ versus $\theta_{3}$ for the spherical silica bead. The gray scale for $n_{1}$ is from 1.33 to 1.40 . The gray scale for $h$ is from 10 to $290 \mathrm{~nm}$, with regions where $n_{1}<1.345$ "blued out". These panels are more magnified than those of Figures 7 and 8 . Scale bar is $3 \mu \mathrm{m}$.

index and the separation distance of a cell sample at the substrate. The resolution is somewhat reduced because the full objective aperture is not used, and scattering produces artifacts. But the approach is very sensitive to the variables it measures, and it does not require multiple colors or changes of medium refractive index to produce useful results.

One main challenge in practice is proper calibration. This is because the results are extremely sensitive to incidence angle (which is why the technique works) but unfortunately the incidence angle is not as well known as might be desired. In addition, the incidence angle usually has a spread of a couple of degrees. This uncertainty is not a big problem in qualitative fluorescence-based TIRF; it only makes the evanescent wave depth somewhat less certain. (The largest uncertainty in TIRF evanescent field depth arises from lack of information about the refractive index of the sample, which the present technique measures directly.) But the intensity of the reflected beam intensity, as measured here, is extremely sensitive to incidence angle in the region just below the critical angle. Therefore, it is essential that, for every set of runs on an unknown cell sample, calibration runs are also performed on known refractive index samples with exactly the same optical alignment.

The theory used to fit the experimental data is itself an approximation, First, the theory is valid only for a system of three discrete planar layers with semi-infinite regions of indices $n_{1}$ and $n_{3}$ sandwiching a finite thickness $n_{2}$. In a real cell sample, $n_{1}$ could be an (unknown) function of $x, y$, and $z$, and also $n_{2}$ could be different to what is assumed in the simple theory. Even a four-layer planar model would introduce two more free parameters (such as the thickness and index of the fourth layer) that, given the uncertainties due to scattering and interference, could not be reliably deduced. Second, the theory (presented explicitly here for p-pol and for s-pol) was applied in its unpolarized (average) form although the laser's illumination was p-pol. However, polarization exerts no influence at all on the TIR critical angle and only a very minor influence in the immediately subcritical range employed here, much smaller than the uncertainties introduced by scattering and interference.

The lateral resolution appears to be no better than about $1 \mu \mathrm{m}$, so small sub-wavelength structures such as mitochondria or secretory vesicles cannot be imaged by the present technique. However, the general theoretical question of how lateral structures of varying refractive index affect near critical angle reflection (and also how they scatter evanescent light) needs future attention. Sparsely-distributed subwavelength structures probably would have a very small effect on reflection, but they might cause scattered light to propagate into angles different from the path of reflected beam, and thereby become observable by an optical arrangement analogous to dark field.

Within the laterally featureless three-layer model, the thinnest intermediate layer that significantly affects the reflected $I$ versus $\theta_{3}$ curve depends on the set of three refractive indicies. Of practical interest is the situation where the intermediate layer is the plasma membrane, sandwiched between a glass substrate and the cell interior. Assuming a refractive index of $n_{2}=1.48$, as reported for a lipid monolayer (Kienle et al., 2014) and a thickness of $h=8 \mathrm{~nm}$, a cell interior of $n_{1}=1.38$ and a glass substrate with $n_{3}=1.52$, the equations predict that the plasma membrane would have virtually no effect on $I$ versus $\theta_{3}$. In other words, the presence of the plasma membrane should have no measurable effect on the imaging of $n_{1}$ as described here. From a physical viewpoint, one reason for the insensitivity of $I$ versus $\theta_{3}$ to the plasma membrane is its extreme sub-wavelength thinness; this would be true regardless of its refractive index. But another more important factor is at play: in the special case of $n_{2}>n_{1}$ (as is the case for the plasma membrane versus cell cytoplasm), as $\theta_{3}$ increases, TIR still occurs first (i.e., at lower $\theta_{3}$ ) at the $n_{2}: n_{1}$ interface. In this special case, an intermediate layer $n_{2}$ would have no impact on the TIR critical angle of the whole three-layer system and only a small effect on the subcritical $I$ versus $\theta_{3}$, regardless of the thickness of $n_{2}$. For the same reasons, introduction of a fourth layer consisting of a high index thin plasma membrane should make virtually no difference to reflected $I$ versus $\theta_{3}$ curve.

Even with the simple three-layer model, it is still possible to obtain a spatially resolved map of $n_{1}$ and $h$ with entirely reasonable values. But a limitation on the accuracy is scattering and interference effects, which cause spurious fluctuations in $I$ versus $\theta_{3}$, and also light returning toward the detector even at incidence angles where it should not be present. These effects are probably not severe on flat biological cells: according to Brunstein et al. (2014), detectable scattering from cells is considerably less than scattering in the optical system itself. But probably on thicker, denser cells, and certainly on dense objects like silica beads, scattering and interference can seriously distort the results, in particular by shifting the evident $n_{1}$ to artifactually lower values. 
The optical setup described here can be reproduced by a simple modification of a commercial TIRF system: the standard filter cube's dichroic mirror need only be replaced by a front surface aluminum film glass mirror extending across a small fraction of the aperture, and the colored filters removed. But further modifications to the system may help ameliorate the scattering and interference artifacts, and possibly make it suitable as a commercial option. Azimuthal scanning of the incident beam direction (Mattheyses et al., 2006) can reduce the apparent effect of flares and shadows on the down-beam side of a sample object, and possibly mathematically analyzed to improve resolution (von Olshausen \& Rohrbach, 2013). Use of an incoherent source (but with a small area emitter such as an LED) should greatly reduce interference fringing. By appropriate blocking at the BFP, it should be possible to observe separately only the scattering image, or only the reflected beam image, and thereby correct the latter with the former. Finally, illumination by a thin semi-circular arc at the BFP (where the radius can be made adjustable to obtain a variable $\theta_{3}$ ) should improve the optical resolution. With these modifications leading to reductions in scattering and interference, it is possible that finer details in complex biological structures can be reliably resolved.

\section{ACKNOWLEDGMENTS}

The author thank Prof. Edward Stuenkel for allowing us to initially set up and test this technique in his lab, and for his comments and help. Supported by NIH RO1-GM110289 to R.W.H. and D.A. and NIH 5T32HL7853-17 to K.P.B.

\section{REFERENCES}

Anantharam, A., Axelrod, D. \& Holz, R.W. (2012). Real-time investigation of fusion pore expansion by imaging of plasma membrane deformations. J Neurochem 122, 661-671.

Axelrod, D. (1981). Zero cost modification of bright field microscopes for imaging phase gradients on cells: Schlieren optics. Cell Biophys 3, 167-173.

Axelrod, D. (2013). Evanescent excitation and emission in fluorescence microscopy. Biophys J 104, 1401-1409. Correction, 104, 2321.

Axelrod, D., Lerner, D. \& SAnds, P.J. (1988). Refractive index within the lens of a goldfish eye determined from the paths of thin laser beams. Vision Res 28, 57-65.

Backman, V., Wallace, M.B., Perelman, L.T., Arendt, J.T., Gurjar, R., Müller, M.G., Zhang, Q., Zonios, G., Kline, E., McGilligan, T., Shapshay, S., Valdez, T., Badizadegan, K., Crawford, J.M., Fitzmaurice, M., Kabani, M.S., Levin, H.S., Seiler, M., Dasari, R.R., ItZKAN, I., VAN DAM, J. \& FELD, M.S. (2000). Detection of preinvasive cancer cells. Nature 406, 35-36.

Bereiter-Hahn, J., Fox, C.H. \& Thorell, B. (1979). Quantitative reflection contrast microscopy of living cells. J Cell Biol 82, 767-779.

Born, M. \& Wolf, E. (1975). Principles of Optics, 5th ed. New York: Pergamon Press. p. 62.

Brunstein, M., Teremetz, M., Herault, K., Tourain, C. \& Oheim, M. (2014). Eliminating unwanted far-field excitation in objectivetype TIRF. Part I. Identifying sources of nonevanescent excitation light. Biophys J 106, 1020-1032.
Cherkezyan, C., Subramanian, H. \& Backman, V. (2014). What structural length scales can be detected by the spectral variance of a microscope image? Opt Lett 39, 4290-4293.

Chew, H., Wang, D.-S. \& Kerker, M. (1979). Elastic scattering of evanescent electromagnetic waves. App Opt 18, 2679-2687.

Choi, W., Fang-Yen, C., Badizadegan, K., OH, S., Lue, N., Dasari, R.R. \& FeLD, M.S. (2007). Tomographic phase microscopy. Nat Methods 4, 717-719.

Curl, C.L., Bellair, C.J., Harris, T., Allman, B.E., Harris, P.J., Stewart, A.G., Roberts, A., Nugent, K.A. \& Delbridge, L.M.D. (2005). Refractive index measurement in viable cells using quantitative phase-amplitude microscopy and confocal microscopy. Cytometry, Part A 65, 88-92.

Farinas, J., Kneen, M., Moore, M. \& Verkman, A.S. (1997). Plasma membrane water permeability of cultured cells and epithelia measured by light microscopy with spatial filtering. J Gen Physiol 110, 283-296.

Friedman, R. \& SHAKed, N.T. (2015). Hybrid reflective interferometric system combining wide-field and single-point phase measurements. IEEE Photonics J 7, 6801413.

Hellen, E.H. \& AXELROD, D. (1987). Fluorescence emission at dielectric and metal-film interfaces. J Opt Soc Am B 4, 337-350.

Hoffman, R. \& Gross, L. (1975). Modulation contrast microscope. Appl Opt 14, 1169-1176.

Holz, R.W. \& AXELROD, D. (2008). Secretory granule behavior adjacent to the plasma membrane before and during exocytosis: Total internal reflection fluorescence studies. Acta Physiol 192, 303-307.

Jafarfard, M.R., Moon, S., Tayebi, B. \& Kim, D.Y. (2014). Dualwavelength diffraction phase microscopy for simultaneous measurement of refractive index and thickness. Opt Lett 39, 2908-2911.

Jenkins, M.H. \& Gaylord, T.K. (2015). Quantitative phase microscopy via optimized inversion of the phase optical transfer function. Appl Opt 54, 8566-8579.

Kienle, D.F., de Souza, J.V., Watkins, E.B. \& Kuhl, T.L. (2014). Thickness and refractive index of DPPC and DPPE monolayers by multiple-beam interferometry. Anal Bioanal Chem 406, 4725-4733.

Kou, S.S., Waller, L., Barbastathis, G. \& Sheppard, C.J.R. (2010). Transport-of-intensity approach to differential interference contrast (TI-DIC) microscopy for quantitative phase imaging. Opt Lett 35, 447-449.

Lee, K., Kim, K., Jumg, J., Heo, J., Cho, S., Lee, S., Chang, G., Jo, Y., PARK, H. \& PARK, Y. (2013). Quantitative phase imaging techniques for the study of cell pathophysiology: From principles to applications. Sensors 13, 4170-4191.

Liu, P.Y., Chin, L.K., Ser, W., Chen, H.F., Hsieh, C.M., Lee, C.H., Sung, K.B., Ayi, T.C., YAP, P.H., Liedberg, B., Wang, K., Bourouina, T. \& Leprince-WAng, Y. (2016). Cell refractive index for cell biology and disease diagnosis: Past, present and future. Lab Chip 16, 634-644.

Loerke, D., Preitz, B., Stuhmer, W. \& Oheim, M. (2000). Super-resolution measurements with evanescent-wave fluorescence excitation using variable beam incidence. J Biomed Opt 5, 23-30.

Martinez-Torres, C., Laperrousaz, B., Berguiga, L., Boyer-Provera, E., Elezgaray, J., Nicolini, F.E., Maguer-Satta, V., Arneodo, A. \& Argoul, F. (2015). Deciphering the internal complexity of living cells with quantitative phase microscopy: A multiscale approach. $J$ Biomed Opt 20, 096005.

Mattheyses, A., SHAW, K.D. \& Axelrod, D. (2006). Effective elimination of laser interference fringing in fluorescence microscopy by spinning azimuthal incidence angle. Microsc Res Tech 69, 642-647. 
McManus, M., Fischbarg, J., Sun, A., Hebert, S. \& Strange, K. (1993). Laser light-scattering system for studying cell volume regulation and membrane transport processes. Am J Physiol 265, C562-C570.

Mitsui, T. (2005). Scanning laser microscope able to detect the refraction of the laser beam. Jpn J Appl Phys 1 Reg Papers Brief Commun Rev Pap 44, 3279-3282.

Neto, J.C., Agero, U., Gazzinelli, R.T. \& Mesquita, O.N. (2016). Measuring optical and mechanical properties of a living cell with defocusing microscopy. Biophys J 91, 1108-1115.

Oheim, M., Loerke, D., Preitz, B. \& Stuhmer, W. (1999). A simple optical configuration for depth-Resolved imaging using variable angle evanescent-wave microscopy. SPIE 3568, 131-140.

Phillips, K.G., Jacques, S.L. \& McCarty, O.J.T. (2012). Measurement of single cell refractive index, dry mass, volume, and density using a transillumination microscope. Phys Rev Lett 109, 118105.

Popescu, G. (2011). Quantitative phase imaging of cells and tissues. New York: McGraw Hill.

Rappaz, B., Barbul, A., Charriere, F., Kuehn, J., Marquet, P., Korenstein, R., Depeursinge, C. \& Magistretti, P. (2007). Erythrocytes volume and refractive index measurement with a digital holographic microscope - art. no. 644509. In Optical Diagnostics and Sensing VII, Coté, G.L. \& Priezzhev, A.V. (Eds.), Proceedings of SPIE 6445, 644509-1-9.

Rappaz, B., Charrière, F., Depeursinge, C., Magistretti, P.J. \& MArquet, P. (2008). Simultaneous cell morphometry and refractive index measurement with dual-wavelength digital holographic microscopy and dye-enhanced dispersion of perfusion medium. Opt Lett 33, 744-746.

Rappaz, B., Marquet, P., Cuche, E., Emery, Y., Depeursinge, C. \& Magistretti, P. (2005). Measurement of the integral refractive index and dynamic cell morphometry of living cells with digital holographic microscopy. Opt Expr 13, 9361-9373.
Ross, K.F.A. (1954). Measurement of the refractive index of cytoplasmic inclusions in living cells by the interference microscope. Nature 174, 836-837.

Steyer, J.A., Horstmann, H. \& Almers, W. (1997). Transport, docking and exocytosis of single secretory granules in live chromaffin cells. Nature 388, 474-478.

Sun, W., Xu, A., MarchuK, K., Wang, G. \& Fang, N. (2011). Whole-cell scan using automatic variable-angle and variableillumination-depth pseudo-total internal reflection fluorescence microscopy. J Lab Autom 16, 255-262.

Tregidgo, C., LevitT, J.A. \& Suhling, K. (2008). Effect of refractive index on the fluorescence lifetime of green fluorescent protein. J Biomed Opt 13, 031218.

Tregidgo, C., Suhling, K. \& Periasamy, A. (2007). Refractive index sensing using fluorescence lifetime imaging (FLIM). Proc SPIE Multiphoton Microsc Biomed Sci VII 6442, 64420W.

Van Manen, H.-J., Verkuijlen, P., Wittendorp, P., Subramaniam, V., VAN DEN Berg, T.K., Roos, D. \& OTto, C. (2008). Refractive index sensing of green fluorescent proteins in living cells using fluorescence lifetime imaging microscopy. Biophys $J$ 94, L67-L69.

Von Olshausen, P. \& Rohrbach, A. (2013). Coherent total internal reflection dark-field microscopy: Label-free imaging beyond the diffraction limit. Opt Lett 38, 4066-4069.

Wick, P.F., Senter, R.A., Parsels, L.A., Uhler, M.D. \& Holz, R.W. (1993). Transient transfection studies of secretion in bovine chromaffin cells and PC12 cells: generation of kainate sensitive chromaffin cells. J Biol Chem 268, 10983-10989.

Yanase, Y., Hiragun, T., Kaneko, S., Gould, H.J., Greaves, M.W. \& Hide, M. (2010). Detection of refractive index changes in individual living cells by means of surface plasmon resonance imaging. Biosens Bioelectron 26, 674-681. 